\title{
An Online Platform for Semantic Validation of UML Models
}

\author{
Marco Brambilla $^{1}$ and Christina Tziviskou ${ }^{2}$ \\ ${ }^{1}$ Politecnico di Milano, Dipartimento di Elettronica ed Informazione, \\ V. Ponzio 34/5, 20133 Milano, Italy \\ marco.brambilla@polimi.it \\ ${ }^{2}$ Università di Bergamo, Ingegneria dell'Informazione e Metodi Matematici, \\ V.le Marconi 5, 24044 Dalmine, Italy \\ christina.tziviskou@unibg.it
}

\begin{abstract}
The Web is becoming the development platform for applications, thus making desktop IDE and CASE tools obsolete. We propose a first example of online support to application designers, consisting in a tool for online validation of UML models based on semantic formalization and reasoning. We base our work on a formalization of the UML models and we exploit Web engineering methods and techniques, applied to Semantic Web technologies, for providing a set of components and patterns that allow management and verification of UML diagrams.
\end{abstract}

Keywords: WebML, UML, conceptual modeling, Semantic Web, reasoner, ontology, validation, class diagram, pattern, component.

\section{Introduction}

The Web is becoming the platform of choice for almost any kind of distributed, as shown by the continuous spreading of online office suites offered as software as a service (SAAS), leading to international web-based collaboration and virtual teamwork. In the medium period the Web will become a platform for the development of applications too: the Eclipse project has transformed the world-famous IDE to the Web platform, and an IBM AlphaWorks project is ongoing to develop a Web browser-based interaction with the Eclipse IDE, under the name EcliFox ${ }^{1}$.

We propose a first example of online support to application designers: a tool for online validation of UML models based on semantic formalization and reasoning. It allows for verification of properties and correctness of UML models. It is used at development time by designers for verifying that the devised conceptual models do not contain inconsistencies, and is also used at runtime once instances of the model are changed, to verify that property correctness still hold for the overall application.

Our choice combines UML class and object diagrams. We formalize them with the DL sROIQ [4], supporting nominals, role inclusion axioms, and transitivity.

\footnotetext{
${ }^{1}$ http://www.alphaworks.ibm.com/tech/eclifox
} 
Reasoning tasks are carried out by Pellet [7], an open-source Java reasoning engine, offering: incremental update of instances, knowledge base debugging, and optimization reasoning techniques. We exploit Web engineering methods, applied to Semantic Web technologies, for providing a set of components and patterns for managing UML diagrams. We adopt WebML [2], a Domain Specific Model for Web application design and development based on data and Semantic Web Services [1]. The WebML specification of a Web application consists of a data schema, and a set of site views, expressing the Web interfaces in terms of pages, which in turn contain units, the atomic publishing primitives that extract contents from the data source. Links between units define the navigation paths and carry data. Updating the underlying data and performing other actions is specified through operation units.

The critical situations of UML models that we resolve with our platform are: unsatisfiable classes, their instantiation would violate some constraints, inconsistent models, contain contradictions among instances and their definitions, and inferred elements, deducted from the modeled elements. These are not automatically identified by traditional type checking tools, leading to possibly system inconsistencies.

Other works have addressed the implementation of automatic tools for UML consistency checking ([3], [6], [8]). They either deal with different type of inconsistencies or they do not provide the means for automatic corrections. In all cases, their reasoning capabilities cannot be integrated automatically in applications.

\section{Web Validation Platform}

The proposed platform mediates between Pellet and the UML developer: it translates UML models to logical axioms in the knowledge base and vice versa, and provides semantic inferences while the developer is unaware of the DL-based mechanisms. Fig. 1 depicts the platform architecture: the Platform Hypertexts (PHs) represent the front-end application accessed by the user for exploring and managing UML models, and the Platform Components (PCs), representing the back-end services for interfacing the front-end with the Knowledge Base (KB).

The components are configurable software artifacts that allow the access and manipulation of UML primitives within the developed application. These primitives refer to a meta-conceptualization as defined by the UML metamodel in [5]: Class,

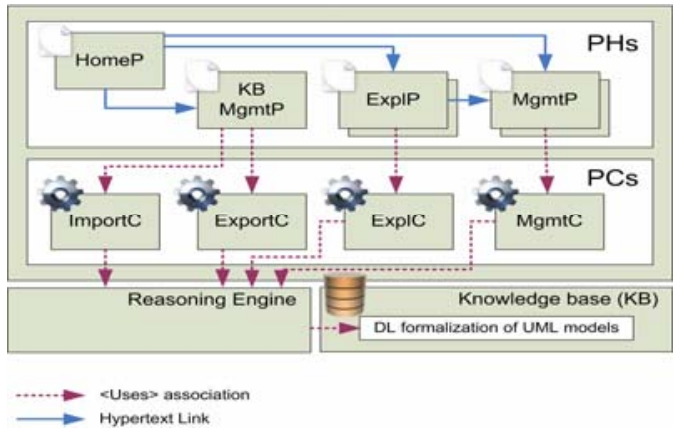

Fig. 1. General architecture of the Web Validation Platform 


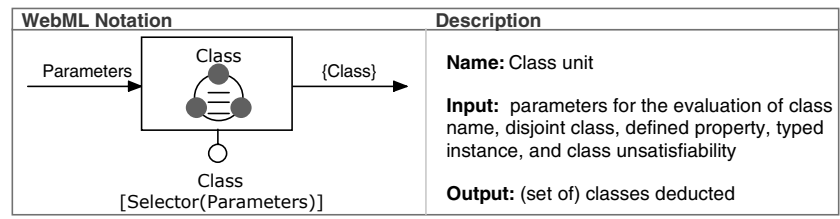

Fig. 2. WebML notation for the semantic Class component

Datatype, Property, Generalization, and Instance. A component is configured upon a UML model. Its execution extracts or modifies UML elements. The Class Component (Fig. 2) defines a set of extraction rules for retrieving UML classes. It explores the DL axioms and deducts classes filtered by selection conditions based upon these axioms.

The components are reusable pieces of code that can be easily combined together and integrated in the WebML specification of applications as predefined patterns to provide a reusable definition of hypertextual solutions for knowledge management. In Fig. 3, we exemplify the usage of "Class and its classification" WebML pattern: (i) the Class Component configured with a Name selection condition retrieves the input class, and (ii) the Class Hierarchy Component configured with a Super.Class condition deducts its ancestor tree. By selecting a more generic class, the Inclusion Explanations page is computed. If the inclusion is asserted in the $\mathrm{KB}$, it is displayed and the user may drop the assertion by navigating to the Remove Component. Otherwise, the assertions causing the inclusion are presented.

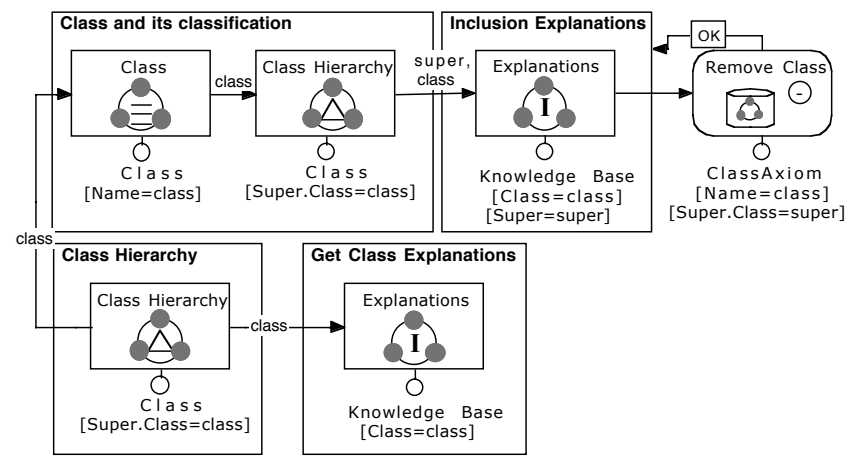

Fig. 3. WebML Class Hierarchy hypertext

\subsection{Wines UML Model Case Study}

To exemplify the usage of the platform, we describe the user navigation upon a Wines UML model, in order to recognize and resolve a class unsatisfiability: the Ice Wine class cannot be instantiated since its instances should have two disjoint parents. The situation becomes known to the developer by exploring the Class Hierarchy page. There, the Ice Wine is presented as unsatisfiable (Fig. 4). By selecting it, the popup window of the Get Class Explanations page appears with the asserted axioms causing the unsatisfiability: Ice Wine has parents the Red Wine and the Dessert Wine, and the 


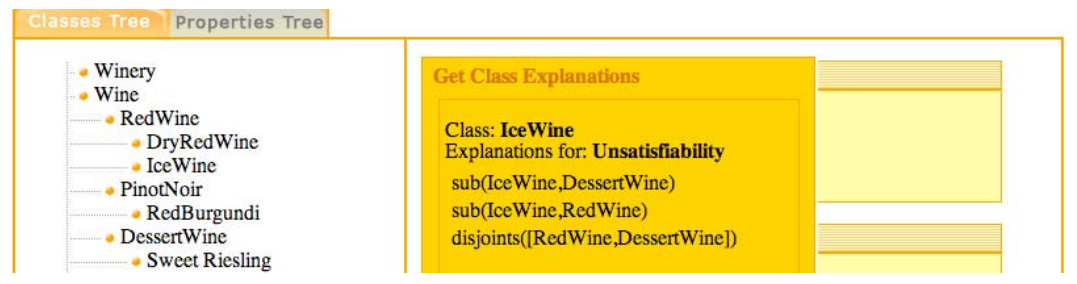

Fig. 4. Explanations pages for the unsatisfiable class Ice Wine

disjoint constraint between them, implies they cannot share instances. A classification can be dropped by the designer invoking the "Class and its classification" pattern.

\section{Conclusions and Future Work}

In this paper we presented a Web platform that allows semantic validation of static UML designs. It is an online tool to be used at development time and at runtime for continuous verification and correctness checking on the application data status.

The resulting Web Platform has three levels of reuse: its Web interfaces can be used for semantic validation of UML models; the patterns can be reused as abstract specifications of exploration and management interfaces for UML models; and the components can be integrated in existing WebML models for providing general purpose reasoning upon the application data semantics.

Future works aim at implementing patterns that resolve the remaining situations in UML models, enable keyword-based search augmenting the flexibility of the user interface, and investigate the use of the components within general-purpose Web applications, to enable them for Semantic Web or Linked Data scenarios.

\section{References}

[1] Brambilla, M., Ceri, S., Facca, F., Celino, I., Cerizza, D., Della Valle, E.: Model-Driven Design and Development of Semantic Web Service Applications. ACM TOIT 8(1) (2007)

[2] Ceri, S., Fraternali, P., Bongio, A., Brambilla, M., Comai, S., Matera, M.: Designing DataIntensive Web Applications. Morgan Kaufmann, San Francisco (2002)

[3] Fillottrani, P., Franconi, E., Tessaris, S.: The new ICOM ontology editor. In: Int.1 W.shop on Description Logics (2006)

[4] Horrocks, I., Kutz, O., Sattler, U.: The Even More Irresistible SROIQ. In: KR 2006, pp. 57-67 (2006)

[5] OMG UML, Unified Modeling Language: Superstructure version 2.1.1. ptc/2007-02-03. OMG document (2007), http: / / www . omg . org / cgi-bin/doc? formal / 07-02-03

[6] Simmonds, J., Bastarrica, C.M.: A tool for automatic UML model consistency checking. In: International Conference on Automated Software Engineering, pp. 431-432 (2005)

[7] Sirin, E., Parsia, B., Grau, B.C., Kalyanpur, A., Katz, Y.: Pellet: A practical OWL-DL reasoner. Journal of Web Semantics 5(2), 51-53 (2007)

[8] Zapata, C.M., Gonzalez, G., Gelbukh, A.: A Rule-Based System for Accessing Consistency Between UML models. In: MICAI Artificial Intelligence, pp. 215-224 (2007) 